\title{
Phytochemical Composition and Antioxidant Activity of Portulaca oleracea: Influence of the Steaming Cooking Process
}

\author{
María del Pilar Fernández-Poyatos, Eulogio J. Llorent-Martínez (D) and Antonio Ruiz-Medina * (D) \\ Department of Physical and Analytical Chemistry, Faculty of Experimental Sciences, University of Jaén, \\ Campus Las Lagunillas, E-23071 Jaén, Spain; mpoyatos@ujaen.es (M.d.P.F.-P.); ellorent@ujaen.es (E.J.L.-M.) \\ * Correspondence: anruiz@ujaen.es; Tel.: +34-953-212-759
}

Citation: Fernández-Poyatos, M.d.P.; Llorent-Martínez, E.J.; Ruiz-Medina, A. Phytochemical Composition and Antioxidant Activity of Portulaca oleracea: Influence of the Steaming Cooking Process. Foods 2021, 10, 94 https://doi.org/10.3390/foods10010094

Received: 16 November 2020 Accepted: 23 December 2020 Published: 5 January 2021

Publisher's Note: MDPI stays neutral with regard to jurisdictional clai$\mathrm{ms}$ in published maps and institutional affiliations.

Copyright: (C) 2021 by the authors. Licensee MDPI, Basel, Switzerland. This article is an open access article distributed under the terms and conditions of the Creative Commons Attribution (CC BY) license (https:// creativecommons.org/licenses/by/ $4.0 /)$

\begin{abstract}
In this work, we compared the phenolic composition and antioxidant capacity of methanolic extracts of raw and steamed aerial parts of Portulaca oleracea L. Two new cyclo-dopa amides were identified, named oleraceins $X$ and $Y$, along with six known ones (oleraceins $A, B, C, N$, J, and U). Compounds identification and quantification were done by high-performance liquid chromatography with diode array and mass spectrometry detections. The most abundant compounds were phenolic alkaloids (oleraceins), and the main quantified compounds were isocitric and citric acids, with concentrations of 500-550 and 440-600 mg/100 g dried extract, respectively. The study of both the influence of the steaming process in Portulaca oleracea L. and total phenolic content and radical scavenging assays (ABTS ${ }^{+}$and DPPH) were also carried out. The total individual phenolic content of raw Portulaca decreased from $1380 \mathrm{mg} / 100 \mathrm{~g}$ DE to $1140 \mathrm{mg} / 100 \mathrm{~g}$ DE after the steaming process. The antioxidant capacity in ABTS and DPPH assays decreased approximately 50 and $40 \%$, respectively, after samples were cooked by steaming. The raw extracts presented the highest concentration of bioactive compounds, as well as higher antioxidant and radical scavenging values.
\end{abstract}

Keywords: Portulaca oleracea; purslane; oleracein; alkaloid; antioxidant; phytochemical; HPLC-MS

\section{Introduction}

Vegetables are sources of antioxidants, minerals, and vitamins, and their consumption can provide a balanced and healthy diet. They are associated with the prevention and reduction of cancer, cardiovascular and other chronic diseases. Phytochemical compounds such as phenolics are some of the contributors to these health benefits [1]. When studying the potential health benefits of foods, including vegetables, it is important to evaluate not only the composition of raw food, but also that of cooked foods, including complex food dishes [2,3].

Portulaca oleracea L., commonly called purslane, belongs to the genus Portulaca from the family Portulacaceae. Purslane is a well-known edible annual herb that grows in Europe, Africa, North America, Australia, and Asia, considering it as an invasive species by their widespread distribution [4]. It grows in orchards, gardens, crop fields, even in roadsides. They are succulent, up to $30 \mathrm{~cm}$ high. The plant exhibits small leaves, with oval shape and dark green color; its stems are reddish and have many small yellow flowers during May to September in which the interior contains tiny black seeds [5] (Figure 1).

With regards to its health benefits, Portulaca oleracea has been called "vegetable for long life" in the Chinese medicinal culture of plants [6], and it is used in folk and traditional medicine as a remedy for many ailments, including dermatitis, abdominal pain, headache, inflammation, intestinal worms, high fever, urinary tract infections, etc., due to possessing a large spectrum of pharmacological effects (analgesic, antioxidant, anti-inflammatory, bactericide, hypocholesterolemic, and hypoglycemic) [7-9]. This plant also contains a high level of proteins, sterols, carotenoids, and polysaccharides [10-12]. It is an important source of omega-3 fatty acids $[13,14]$. Moreover, it has a wide range of vitamins $(\mathrm{A}, \mathrm{C}, \mathrm{E}$, and some of complex-B) [10,12] and minerals ( $\mathrm{Ca}, \mathrm{Fe}, \mathrm{Mn}, \mathrm{P}$, and Se) [12,15]. In addition, the 
determination of volatile compounds, amino acids, antimicrobial assays [10], as well as tocopherols, organic acids, and cytotoxicity tests [11] have been carried out in studies of Portulaca oleracea.
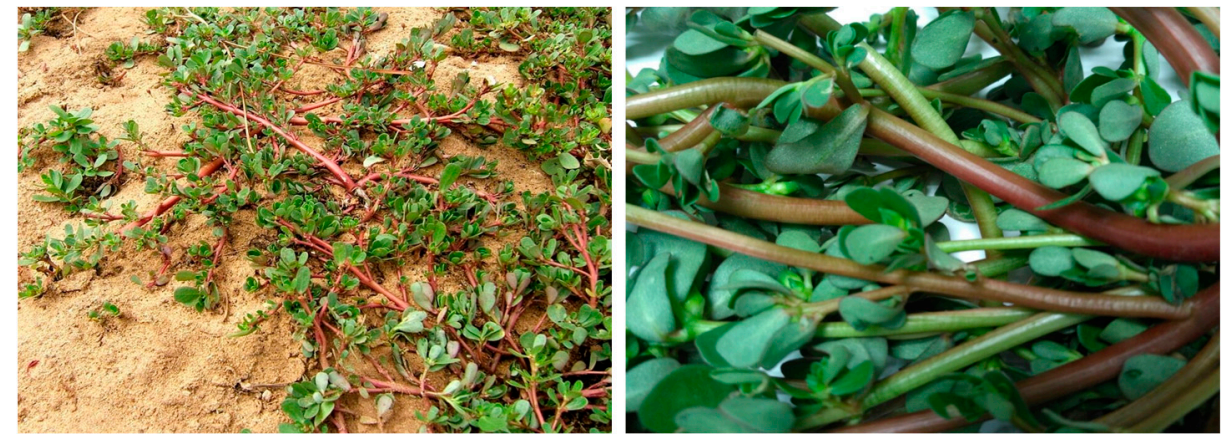

Figure 1. Portulaca oleracea L.

Purslane is rich in polyphenols, which includes phenolic acids and flavonoids. Several reports on raw Portulaca oleracea have been made about its phenolic composition, total phenolic, and total flavonoid contents. Compounds such as caffeic acid, ferulic acid, kaempferol, quercetin, and rutin have been previously identified [10-14,16-18]. This plant also shows a class of alkaloids, which are secondary metabolites, named cyclo-dopa amides, or oleraceins, are increasing the general interest $[12,13,19]$. These compounds show more or similar potent antioxidant characteristics than some natural antioxidants such as vitamin $\mathrm{C}$ and vitamin $\mathrm{E}[12,13]$.

Portulaca oleracea is consumed both raw and cooked. It is an important component of green salad and its leaves and stems are used raw, alone, or with other vegetables. Fresh juices are also prepared, and purslane is commonly added to soups, steamed, in teas. or used as potherb $[5,10,12,13,20]$. The impact of cooking treatment on vegetables can cause changes in nutritional composition and levels of bioactive compounds [21]. Studies on the effects on cooking have been carried out in Portulaca oleracea, observing differences according to the type of cooking, but mainly focus on boiling and bleaching, or cooked with some food additives such as lemon juice or vinegar [22-24].

This work aimed to determine the composition of the phenolic content of raw and steamed aerial parts of Portulaca oleracea, as well as its antioxidant activity, thus evaluating the effect of the steaming cooking process, which has not been reported so far. In addition, the structure of two new oleraceins has been elucidated. The hypothesis was that the steaming process would decrease both phenolic composition and antioxidant activity when compared to raw Portulaca samples, but still maintaining a reasonable number of bioactive compounds.

\section{Materials and Methods}

\subsection{Chemicals and Reagents}

All reagents and standards were of analytical reagent grade. Standards of caffeic acid, catechin, citric acid, ferulic acid, hydroxytyrosol, kaempferol, quercetin, and sinapic acid were purchased from Sigma-Aldrich (Madrid, Spain). All solutions were prepared in methanol (MeOH) HPLC-grade (Sigma-Aldrich). LC-MS grade acetonitrile (Panreac; Barcelona, Spain) and ultrapure water (Milli-Q Waters purification system; Millipore; Milford, MA, USA) were also used.

Ethanol (96\%), Folin-Ciocalteu's phenol reagent (FCR) and sodium carbonate $\left(\mathrm{Na}_{2} \mathrm{CO}_{3}\right)$ were purchased from Panreac (Madrid, Spain). 2,2'-azinobis (3-ethylbenzthiazoline-6sulfonic acid) (ABTS; $\geq 98 \%$ ), 2,2-diphenyl-1-picrylhydrazyl (DPPH; $95 \%$ ), gallic acid monohydrate (>98\%), 6-hydroxy-2,5,7,8-tetramethylchroman-2-carboxylic acid (Trolox; 97\%), formic acid $(98-100 \%)$, and potassium persulfate $(>99 \%)$ were obtained from Sigma-Aldrich. 


\subsection{Sample Preparation and Extraction}

Portions of fresh aerial parts of different Portulaca oleracea plants were randomly and carefully collected, approximately $1000 \mathrm{~g}$, in an orchard in Andújar (southeast of Spain; $38^{\circ} 03^{\prime} 40.7^{\prime \prime} \mathrm{N} 4^{\circ} 02^{\prime} 58.4^{\prime \prime} \mathrm{W}, 235 \mathrm{~m}$ a.s.l.), in October 2019. Botanical authentication was carried out by the botanist Dr. Carlos Salazar Mendías (Department of Animal Biology, Plant Biology and Ecology of the University of Jaén, Spain). Only the freshest samples were selected. They were mixed into the same pool, washed with Milli-Q water, and cut into small portions (approximately 10-cm-long). A portion of fresh (raw) Portulaca oleracea was analyzed to check the initial phenolic content and antioxidant capacity, whereas another portion was steamed. The last one was cooked in a traditional stainless-steel steamer (three pieces: A pot, a steamer basket, and a lid) for $5 \mathrm{~min}$. Cooking time was selected according to the usual time used in domestic recipes.

Previous works compared different extracting solvents (methanol, ethanol, ethyl acetate, and water) for the analysis of Portulaca composition, reporting that methanol was the most suitable one for the extraction of phenolic compounds [25-27]. Hence, we selected $\mathrm{MeOH}$ for the extractions of phenolics from raw and steamed samples.

For both raw and steamed samples, aerial parts were lyophilized (Lyoquest -55 ECO, Telstar; Barcelona, Spain) and crushed with a grinder. Ultrasound-assisted extraction was done by placing $2.5 \mathrm{~g}$ of dry material in $50 \mathrm{~mL} \mathrm{MeOH}$ for $10 \mathrm{~min}$ (Qsonica Sonicators; Newton, CT, USA) with a power of $55 \mathrm{~W}$ and a frequency of $20 \mathrm{kHz}$ (50\% power). Each sample was extracted in triplicate. Then, solutions were filtered through Whatman No.1 filters and the solvent was evaporated under reduced pressure in a rotary evaporator at $40{ }^{\circ} \mathrm{C}$. Dried extracts (DE) were stored at $-20{ }^{\circ} \mathrm{C}$ until analysis. A scheme of the process is shown in Figure 2.

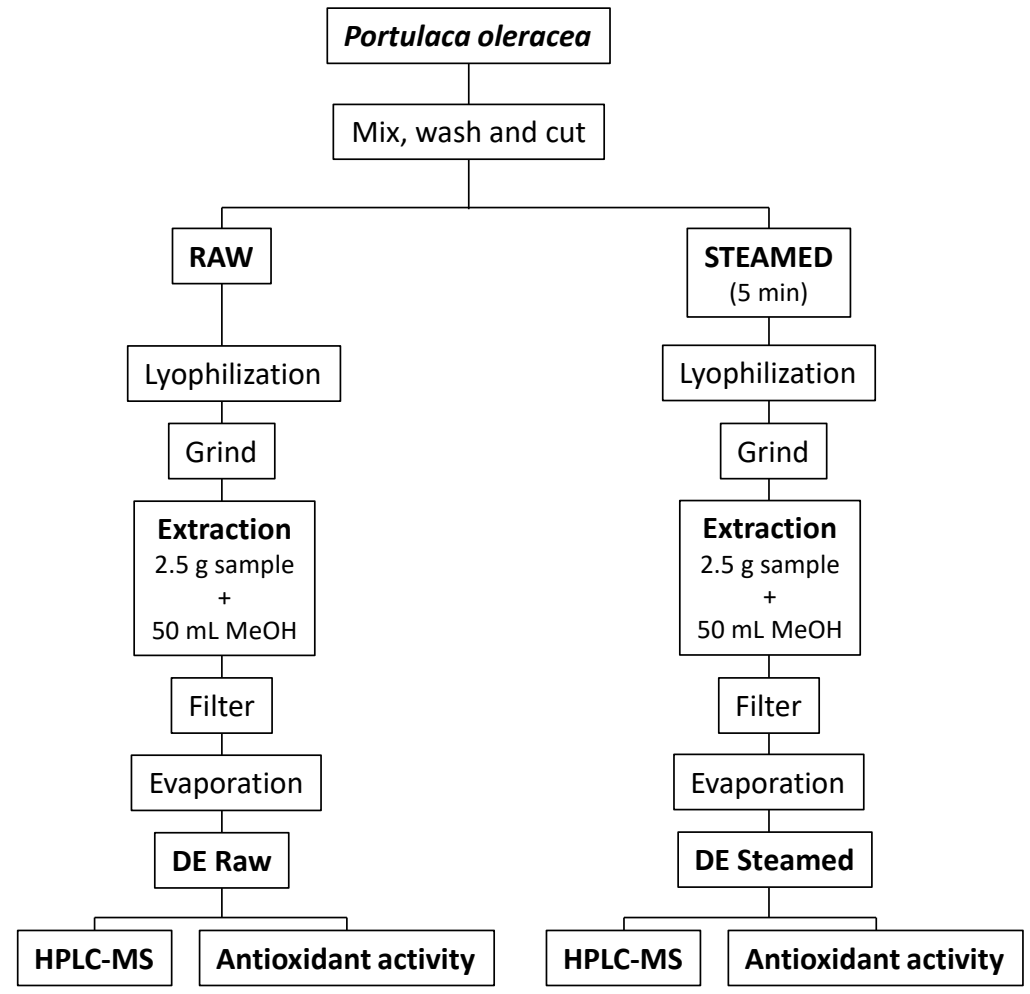

Figure 2. Scheme of the experiments. DE: Dried extract. 


\subsection{HPLC Analysis}

The raw and steamed extracts were analyzed by high-performance liquid chromatography with diode-array and mass spectrometry detection in an Agilent Series 1100 with a G1315B diode array detector and an ion trap mass spectrometer (Esquire 6000, Bruker Daltonics) with an electrospray interface. Detail conditions are given in [28]. We also used an Agilent 1200 HPLC equipped with an Agilent 6530B quadrupole-time-of-flight mass spectrometer (Q-TOF MS) to confirm the identity of the compounds by exact mass determination.

Calibration curves for caffeic acid, catechin, citric acid, ferulic acid, hydroxytyrosol, kaempferol, quercetin, and sinapic acid at concentrations 1-100 $\mu \mathrm{g} \mathrm{mL}^{-1}$ in MeOH. Chromatograms were recorded at $280 \mathrm{~nm}$ for catechin and hydroxytyrosol; $320 \mathrm{~nm}$ for caffeic acid, ferulic acid, and sinapic acid; and $350 \mathrm{~nm}$ for kaempferol and quercetin. For citric acid, the instrument was operated in product ion scan MS/MS mode with the selected MS/MS transition $191 \rightarrow 111$, and voltage amplitude of $0.6 \mathrm{~V}$.

\subsection{Assay for Total Phenolic Content and Determination of Antioxidant Capacity}

For the following analyses, the procedures were adapted from [29]. Total phenolic content (TPC) and antioxidant assays were carried out using a UV-Vis Spectrophotometer (Zuzi Spectrophotometer, model 4201/50; Beriain, Navarra, Spain). For TPC, 3-5 mg mL ${ }^{-1}$ of dried extracts were analyzed, and the obtained results are reported as $\mathrm{mg}$ of gallic acid equivalents (GAE) per g of dried extract. Regarding the antioxidant capacity, two different spectrophotometric radicals scavenging tests $\left(\mathrm{ABTS}^{+}\right.$and DPPH). Sample solutions of $0.15 \mathrm{mg} \mathrm{mL}^{-1}$ and $1 \mathrm{mg} \mathrm{mL}^{-1}$ in $\mathrm{MeOH}$ were prepared for $\mathrm{ABTS}^{+}$and DPPH assays, respectively. Results are expressed as $\mu \mathrm{mol}$ of Trolox equivalents (TE) per g of dried extract, for both assays. All assays were done in triplicate.

\section{Results and Discussion}

In this work, we compared the composition of the aerial parts of Portulaca oleracea in its raw and steamed form. The most common cooking methods for this plant are boiling and steaming. Previous studies on other vegetables, such as pumpkin, reported steaming as better than boiling regarding antioxidant activity and phenolic content [30]; similarly, a study performed on cauliflower reported that due to the degree of exposure to heat and water (different wet-thermal process cooking), steaming induced the least reductive effect on phytochemical components [31]. We thus studied the effect of steaming on the antioxidant and phenolic composition of Portulaca oleracea.

\subsection{HPLC-MS Analysis of Raw and Steamed Extracts}

Compounds characterization was carried out by mass spectrometry, using both negative and positive ion modes, although most of the compounds were identified in negative mode (Table 1). The base peak chromatograms of raw and steamed extracts of Portulaca oleracea aerial parts are shown in Figure 3. We identified or tentatively characterized 24 compounds; almost $30 \%$ were phenolic acids and $25 \%$ flavonoids. There was a higher percentage (33\%) that corresponded with alkaloid compounds, specifically oleraceins.

Table 1. Characterization of phytochemicals found in extracts raw and steamed of Portulaca oleracea by HPLC-DAD/ESI-MSn

\begin{tabular}{ccccc}
\hline No. & $\begin{array}{c}\mathbf{t}_{\mathbf{R}} \\
(\mathbf{m i n})\end{array}$ & $\begin{array}{c}{\left[\mathbf{M}-\mathbf{H}^{-}\right.} \\
\mathbf{m} / \boldsymbol{z}\end{array}$ & $m / z \mathbf{~ \% ~ B a s e ~ P e a k )}$ & Assigned Identification \\
\hline 1 & 1.8 & 191 & $\mathrm{MS}^{2}[191]: 173(41), 111(100)$ & Isocitric acid \\
2 & 2.6 & 191 & $\mathrm{MS}^{2}[191]: 173(80), 111(100)$ & Citric acid * \\
3 & 3.6 & 315 & $\mathrm{MS}^{2}[315]: 153(100), 135(52)$ & Hydroxytyrosol hexoside \\
\end{tabular}


Table 1. Cont.

\begin{tabular}{|c|c|c|c|c|}
\hline No. & $\begin{array}{c}t_{R} \\
(\min )\end{array}$ & $\begin{array}{l}{[\mathrm{M}-\mathrm{H}]^{-}} \\
\mathrm{m} / z\end{array}$ & $m / z$ (\% Base Peak) & Assigned Identification \\
\hline 4 & 4.4 & 371 & $\begin{array}{c}\mathrm{MS}^{2} \text { [371]: } 353(18), 209(100), 191(44) \\
\mathrm{MS}^{3}[371 \rightarrow 209]: 191(100) \\
\mathrm{MS}^{4}[371 \rightarrow 209 \rightarrow 191]: 129(100)\end{array}$ & Caffeoylglucaric acid \\
\hline 5 & 5.2 & 355 & $\begin{array}{c}\mathrm{MS}^{2} \text { [355]: } 209(29), 191(100) \\
\mathrm{MS}^{3}[355 \rightarrow 191]: 149(94), 129(100)\end{array}$ & Caffeic acid glucuronide isomer \\
\hline 6 & 5.8 & 341 & $\begin{array}{c}\operatorname{MS}^{2}[341]: 179(100), 161(23), 135(22) \\
\operatorname{MS}^{3}[341 \rightarrow 179]: 135(100)\end{array}$ & Caffeic acid-O-hexoside \\
\hline 7 & 6.7 & 355 & $\mathrm{MS}^{2}$ [355]: 337 (11), 209 (34), 191 (100), 129 (4) & Caffeic acid glucuronide isomer \\
\hline 8 & 8.6 & 289 & $\begin{array}{c}\mathrm{MS}^{2} \text { [289]: } 245 \text { (100), } 205 \text { (25), } 203 \text { (12), } 179 \text { (17) } \\
\mathrm{MS}^{3} \text { [289-245]: } 203(100), 175(45), 159(41), 123(50)\end{array}$ & Catechin * \\
\hline 9 & 9.8 & 355 & $\begin{array}{l}\mathrm{MS}^{2} \text { [355]: } 193(100), 178(9), 134(12) \\
\operatorname{MS}^{3}[355 \rightarrow 193]: 178(100), 149(42)\end{array}$ & Ferulic acid-O-hexoside \\
\hline 10 & 10.4 & 664 & $\begin{array}{c}\operatorname{MS}^{2}[664]: 502(100) \\
\operatorname{MS}^{3}[664 \rightarrow 502]: 340(100)\end{array}$ & Oleracein C \\
\hline 11 & 10.7 & 385 & $\begin{array}{c}\mathrm{MS}^{2} \text { [385]: } 247(45), 223(100), 205(57) \\
\left.\mathrm{MS}^{3} \text { [385 } \rightarrow 223\right]: 208(20), 179(13), 164(100), 149(13) \\
\text { MS }^{4}[385 \rightarrow 223 \rightarrow 164]: 149(100)\end{array}$ & Sinapic acid-O-hexoside \\
\hline 12 & 11.6 & 289 & $\begin{array}{l}\mathrm{MS}^{2} \text { [289]: } 245 \text { (100), } 205(45), 203(22), 179(18) \\
\mathrm{MS}^{3}[289 \rightarrow 245]: 217(89), 203(100), 161(55)\end{array}$ & Epicatechin \\
\hline 13 & 11.9 & 680 & $\begin{array}{l}\mathrm{MS}^{2} \text { [680]: } 518(100), 356(59), 246(28), 202(18) \\
\operatorname{MS}^{3}[680 \rightarrow 518]: 356(100), 246(37), 202(14) \\
\operatorname{MS}^{4}[680 \rightarrow 518 \rightarrow 356]: 246(100), 202(32)\end{array}$ & Oleracein X \\
\hline 14 & 15.1 & 518 & $\begin{array}{c}\mathrm{MS}^{2}[518]: 356(100), 246(25), 202(8) \\
\mathrm{MS}^{3}[518 \rightarrow 356]: 246(100), 202(28), 150(10) \\
\mathrm{MS}^{4}[518 \rightarrow 356 \rightarrow 246]: 202(100)\end{array}$ & Oleracein Y \\
\hline 15 & 18.5 & 502 & $\begin{array}{c}\mathrm{MS}^{2} \text { [502]: } 340(100), 252(13), 145(3) \\
\mathrm{MS}^{3}[502 \rightarrow 340]: 296(65), 252(100), 194(47), 145(87)\end{array}$ & Oleracein A \\
\hline 16 & 19.7 & 532 & $\begin{array}{l}\mathrm{MS}^{2}[532]: 370(100), 326(6), 282(9), 175(4) \\
\mathrm{MS}^{3}[532 \rightarrow 370]: 282(100), 161(72), 175(63)\end{array}$ & Oleracein B \\
\hline 17 & 19.7 & 309 & $\begin{array}{l}\mathrm{MS}^{2} \text { [309]: } 193(100), 134(5) \\
\mathrm{MS}^{3}[309 \rightarrow 193]: 149(100), 134(80)\end{array}$ & Ferulic acid derivative \\
\hline 18 & 20.5 & 463 & $\begin{array}{c}\mathrm{MS}^{2} \text { [463]: } 301(100), 271(5), 179(4), 151(4) \\
\mathrm{MS}^{3}[463 \rightarrow 301]: 271(100), 255(43), 229(51), 179(96), 151(43)\end{array}$ & Quercetin-O-hexoside isomer \\
\hline 19 & 21.1 & 840 & $\begin{array}{l}\mathrm{MS}^{2}[840]: 694(100), 664(77), 518(61) \\
\operatorname{MS}^{3}[840 \rightarrow 664]: 518(100), 340(58)\end{array}$ & Oleracein $\mathrm{N}$ \\
\hline 20 & 22.7 & $342(+)$ & $\begin{array}{c}\mathrm{MS}^{2}[342]: 177(22), 147(100), 119(14) \\
\operatorname{MS}^{3}[342 \rightarrow 147]: 119(100)\end{array}$ & Oleracein U \\
\hline 21 & 24.0 & 856 & $\begin{array}{c}\mathrm{MS}^{2}[856]: 694(100), 680(16), 356(35), 246(14) \\
\operatorname{MS}^{3}[856 \rightarrow 694]: 356(100), 246(81)\end{array}$ & Oleracein J \\
\hline 22 & 24.0 & 447 & $\begin{array}{c}\mathrm{MS}^{2} \text { [447]: } 285 \text { (100), } 284(76), 255(45) \\
\mathrm{MS}^{3}[447 \rightarrow 284]: 257(24), 255(100), 227(37)\end{array}$ & Kaempferol-O-hexoside \\
\hline 23 & 24.5 & 477 & $\begin{array}{c}\mathrm{MS}^{2} \text { [477]: } 357(11), 315(100), 285(31), 271(19), 151(8) \\
\left.\text { MS }^{3} \text { [477 } \rightarrow 315\right]: 300(27), 285(100), 271(69) \\
\text { MS }^{4}[477 \rightarrow 314 \rightarrow 285]: 271(100)\end{array}$ & Isorhamnetin-O-hexoside \\
\hline 24 & 27.0 & 463 & $\begin{array}{l}\operatorname{MS}^{2}[463]: 301(100), 179(3), 151(2) \\
\operatorname{MS}^{3}[463 \rightarrow 301]: 179(100), 151(41) \\
\operatorname{MS}^{4}[463 \rightarrow 301 \rightarrow 179]: 151(100)\end{array}$ & Quercetin-O-hexoside isomer \\
\hline 25 & 29.8 & 677 & $\begin{array}{l}\mathrm{MS}^{2} \text { [677]: } 659(42), 645(100), 627(30), 617(64), 585(22) \\
\mathrm{MS}^{3}[677 \rightarrow 645]: 627(80), 541(73), 489(100), 462(78) \\
\quad \mathrm{MS}^{4}[677 \rightarrow 645 \rightarrow 489]: 462(49), 445(53), 417(100)\end{array}$ & Unknown \\
\hline
\end{tabular}

* Identified with analytical standards. 


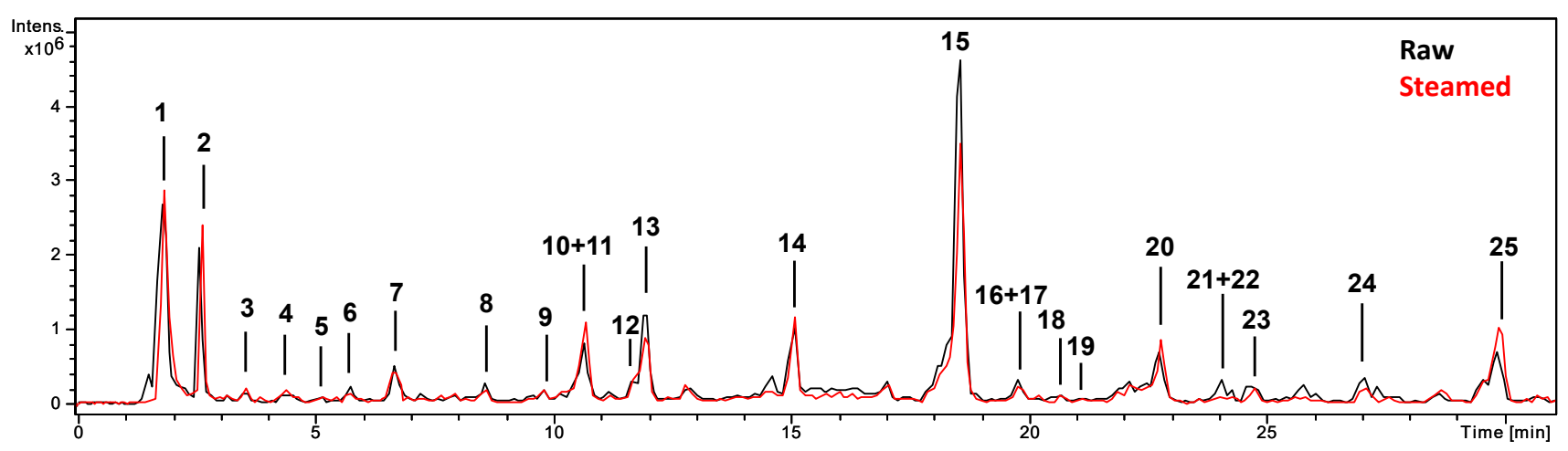

Figure 3. HPLC-ESI/MS ${ }^{n}$ base peak chromatograms (BPC) of the raw and steamed extracts of Portulaca oleracea aerial parts.

\subsubsection{Phenolic Acids}

Compound 4 displayed deprotonated molecular ion at $\mathrm{m} / \mathrm{z} 371$ and fragment ions $m / z 353,209,191$, and 129. This fragmentation pattern has been reported in the bibliography for both caffeoylglucaric acid and hydroxyferulic acid-hexoside. Hence, Q-TOF was used to calculate the exact mass, and the experimental fragmentation pattern was compared with the database METLIN. Compound 4 was thus unequivocally identified as caffeoylglucaric acid. In the same way, compounds $\mathbf{5}$ and $\mathbf{7}$ were identified as caffeic acid glucuronide isomers.

Compounds 9 and 17 were ferulic acid derivatives. Compound $\mathbf{9}$, with $[\mathrm{M}-\mathrm{H}]^{-}$at $\mathrm{m} / \mathrm{z}$ 355 , suffered the neutral loss of a hexoside (162 Da) yielding the $\mathrm{MS}^{2}$ base peak at $\mathrm{m} / \mathrm{z} 193$ and the fragment ions characteristic of ferulic acid at $\mathrm{m} / \mathrm{z} 178,149$ and 134; this compound was characterized as ferulic acid-O-hexoside [32]. Compound 17 exhibited [M-H] ${ }^{-}$ion at $\mathrm{m} / \mathrm{z} 309$ and was tentatively characterized as ferulic acid derivative due to the fragment ions at $m / z$ 193, 149 and 134 (typical of ferulic acid).

Compound 6 was identified as caffeic acid-O-hexoside. It presented $[\mathrm{M}-\mathrm{H}]^{-}$ion at $\mathrm{m} / \mathrm{z}$ 341 , produced a fragment ion at $\mathrm{m} / \mathrm{z} 179$ (caffeic acid) due to the neutral loss of a hexoside, and typical caffeic acid fragments at $m / z 161$ and 135 [29]. Compound 11 showed [M-H] ${ }^{-}$ ion at $m / z 385$. This compound was characterized as a hydroxycinnamoyl glycoside, namely sinapic acid-O-hexoside or sinapoyl hexoside. The base peak fragment ion in $\mathrm{MS}^{2}$ was at $\mathrm{m} / \mathrm{z} 223$ (neutral loss of $162 \mathrm{Da}$, corresponding to a hexoside molecule). Other fragment ions were at $\mathrm{m} / \mathrm{z} 205$ (loss of water, $18 \mathrm{Da}$ ), $\mathrm{m} / \mathrm{z} 179$ (loss of $44 \mathrm{Da}$, carbon dioxide), and $\mathrm{m} / \mathrm{z}$ 149 (30 Da, generated by the losses of two $\mathrm{CH}_{3}$ radicals) [33].

\subsubsection{Flavonoids}

Compounds 8 and 12 displayed $[\mathrm{M}-\mathrm{H}]^{-}$at $m / z 289$ and identical fragmentation patterns $(\mathrm{m} / \mathrm{z} 245,205,203$, and 179). These compounds were identified as catechin and epicatechin, respectively, considering that epicatechin elutes after catechin in reversedphase column [34].

Compounds 18 and 24 exhibited [M-H] ${ }^{-}$ions at $m / z 463$ and suffered the neutral loss of $162 \mathrm{Da}$ (hexoside), producing quercetin at $\mathrm{m} / \mathrm{z} 301$ (characteristic fragments at $\mathrm{m} / \mathrm{z} 179$ and 151), so both compounds were identified as quercetin-O-hexoside isomers [32].

Compound 22 was named as kaempferol-O-hexoside. It showed $[\mathrm{M}-\mathrm{H}]^{-}$ion at $\mathrm{m} / \mathrm{z}$ 447 and the neutral loss of 162 Da yielded the aglycone kaempferol at $m / z$ 285/284 [35].

Compound 23 was characterized as isorhamnetin-O-hexoside, presenting the aglycone at $m / z 315$, due to the neutral loss of a hexose molecule, and typical fragment ion of isorhamnetin at $m / z 300$ [34].

\subsubsection{Alkaloids}

Compounds 10, 13, 14, 15, 16, 19, 20, and 21 were characterized as cyclo-dopa amides, named oleraceins. In previous works, the presence of these phenolic alkaloids has been 
reported in Portulaca oleracea [19,36,37]. Compound 10 was identified as oleracein C, with $[\mathrm{M}-\mathrm{H}]^{-}$at $m / z 664$ and fragment ions at $m / z 502\left([\mathrm{M}-\mathrm{Glc}-\mathrm{H}]^{-} ; \mathrm{Glc}=\right.$ glucoside $)$ and 340 $\left([\mathrm{M}-\mathrm{Glc}-\mathrm{Glc}-\mathrm{H}]^{-}\right)$[19]. Compound 13 showed an $[\mathrm{M}-\mathrm{H}]^{-}$ion at $m / z$ 680. This compound has not been previously identified and, according to the study of the fragments, we propose a similar structure to oleracein $\mathrm{J}$ but without the ferulic acid group $\left[\mathrm{M}-\mathrm{C}_{10} \mathrm{H}_{8} \mathrm{O}_{3}-\mathrm{H}\right]^{-}[19]$, naming it as oleracein $X$, following the order of nomenclature of the compounds of this alkaloid family, whose structure was determined as 5-hydroxy-1-caffeic acyl-2,3dihydro$1 \mathrm{H}$-indole-2-carboxylic acid-6-O-diglucoside $\left(\mathrm{C}_{30} \mathrm{H}_{35} \mathrm{NO}_{17}\right)$ (Figure 4). The fragment ions of oleracein $\mathrm{X}$ were $\mathrm{m} / \mathrm{z} 518$ (neutral loss of 163 Da corresponding to a caffeic acyl group $\left.\left(\left[\mathrm{M}-\mathrm{C}_{9} \mathrm{H}_{6} \mathrm{O}_{3}-\mathrm{H}\right]^{-}\right)\right), m / z 356$ (neutral loss of 325 Da belonging to two glucoside molecules $\left.\left(\left[\mathrm{M}-\mathrm{C}_{12} \mathrm{H}_{20} \mathrm{O}_{10}-\mathrm{H}\right]^{-}\right)\right), m / z 246$ (neutral loss of $435 \mathrm{Da}$ corresponding to $325 \mathrm{Da}$ and 110 Da from two glucoside (Glc) molecules and a 1,2-dihydroxybenzene molecule ([M-2Glc$\left.\mathrm{C}_{6} \mathrm{H}_{5} \mathrm{O}_{2}-\mathrm{H}\right]^{-}$)), and $m / z 202$ (neutral loss of 479 Da belonging to $435 \mathrm{Da}$ of the loss explained previously and an additional loss of $44 \mathrm{Da}$ from a carboxylic group ([M-2Glc- $\mathrm{C}_{6} \mathrm{H}_{5} \mathrm{O}_{2}-\mathrm{CO}_{2}-$ $\left.\mathrm{H}^{-}\right)$). The proposed molecular formula for compound $\mathbf{1 3}$ (oleracein $\mathrm{X}$ ) has been confirmed by exact mass using Q-TOF analysis.

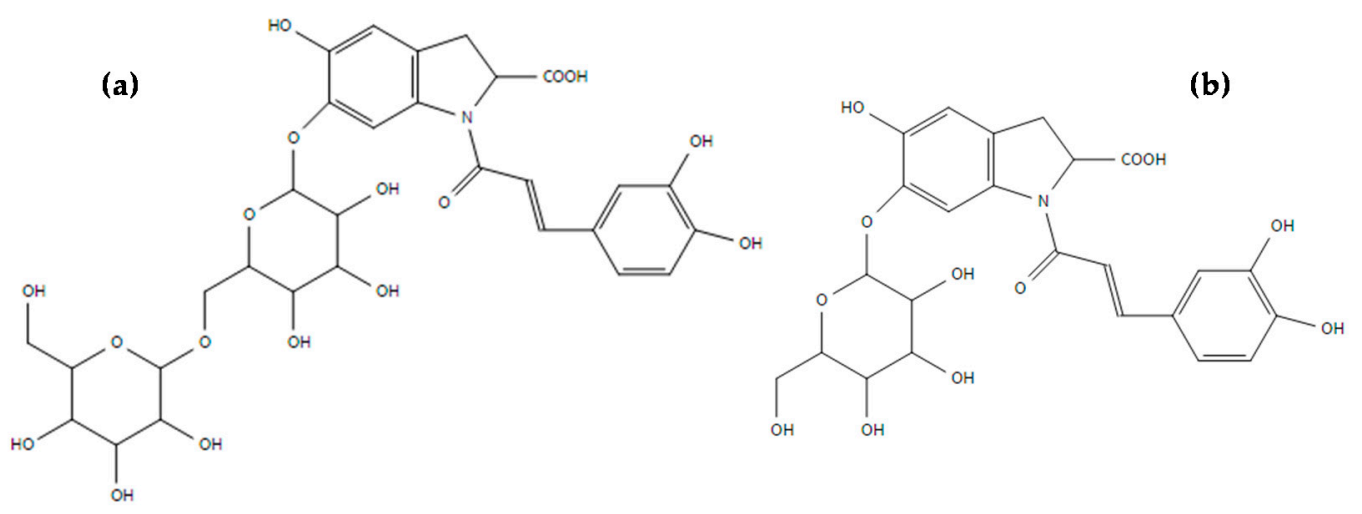

Figure 4. Proposed structures of oleracein $X(\mathbf{a})$ and oleracein $Y(\mathbf{b})$.

Compound 14 showed an $[\mathrm{M}-\mathrm{H}]^{-}$ion at $m / z 518$ and fragment ions at $m / z 356$ (neutral loss of $163 \mathrm{Da}$ corresponding to a glucoside molecule ([M-Glc-H] ${ }^{-}$)), m/z 246 (neutral loss of $110 \mathrm{Da}$ from a 1,2-dihydroxybenzene molecule ([M-Glc- $\left.\mathrm{C}_{6} \mathrm{H}_{5} \mathrm{O}_{2}-\mathrm{H}\right]^{-}$)) and $m / z 202$ (neutral loss of $44 \mathrm{Da}$ from the previous fragment ion due a carboxylic group ([M-Glc$\left.\mathrm{C}_{6} \mathrm{H}_{5} \mathrm{O}_{2}-\mathrm{CO}_{2}-\mathrm{H}^{-}\right)$). This compound has not been previously described and, according to the fragmentation pattern, we propose a new compound called oleracein $\mathrm{Y}$, whose structure was determined as 5-hydroxy-1-caffeic acyl-2,3dihydro- $1 H$-indole-2-carboxylic acid-6-O-glucoside $\left(\mathrm{C}_{24} \mathrm{H}_{25} \mathrm{NO}_{12}\right.$ (Figure 4$)$, similar to oleracein $\mathrm{V}$ structure but without a hydroxyl group $\left(\mathrm{C}_{24} \mathrm{H}_{25} \mathrm{NO}_{13}\right)$ [12]. The molecular formula proposed for compound 14 (oleracein $\mathrm{Y}$ ) was also confirmed by exact mass using Q-TOF results.

Compound 15 was the highest peak in the chromatograms of both raw and steamed extracts. It showed $[\mathrm{M}-\mathrm{H}]^{-}$ion at $m / z 502$ and the characteristic fragmentation pattern of oleracein A $(m / z$ 340, 252, 296) [10]. Compound 16 was identified as oleracein B. It presented an $[\mathrm{M}-\mathrm{H}]^{-}$ion at $\mathrm{m} / z 532$ and the typical fragmentation of this alkaloid $(\mathrm{m} / \mathrm{z} 370$, 326,282 ) [10]. Compound 19 was characterized as another cyclo-dopa amide, specifically, oleracein N. It displayed an $[\mathrm{M}-\mathrm{H}]^{-}$ion at $m / z 840$ and the fragment ions characteristics of this alkaloid $(m / z 694,664,518)$ [19]. Compound 20 was tentatively characterized as oleracein $\mathrm{U}$, due to the $[\mathrm{M}+\mathrm{H}]^{+}$ion at $m / z 342$ and base peak at $m / z 147$, previously reported in bibliography [13]. Compound 21 was identified as oleracein J. It showed [M$\mathrm{H}]^{-}$ion at $m / z 856$ and the typical fragments ion of this cyclo-dopa amide $(\mathrm{m} / \mathrm{z} 694,680$, 356, and 246) [19]. In Figure 5, all the oleraceins reported in scientific literature (oleracein A to oleracein $\mathrm{W}$, including the new oleraceins $\mathrm{X}$ and $\mathrm{Y}$ ) have been gathered, showing the chemical structures and molecular ions $[\mathrm{M}-\mathrm{H}]^{-}[12,19,38-40]$. 
<smiles>[R6]Oc1cc2c(cc1O)CC([2H])C(=O)N2C(=O)/C=C/c1cc([R6])c([R5])c([R3])c1[R3]</smiles>

$\begin{array}{llllllll}\text { Oleracein A } & \left(\mathrm{C}_{24} \mathrm{H}_{25} \mathrm{NO}_{11} ; 502\right) & \mathrm{R}_{1}=\mathrm{Glc} & \mathrm{R}_{2}=\mathrm{H} & \mathrm{R}_{3}=\mathrm{H} & \mathrm{R}_{4}=\mathrm{H} & \mathrm{R}_{5}=\mathrm{OH} & \mathrm{R}_{6}=\mathrm{H} \\ \text { Oleracein B } & \left(\mathrm{C}_{25} \mathrm{H}_{27} \mathrm{NO}_{12} ; 532\right) & \mathrm{R}_{1}=\mathrm{Glc} & \mathrm{R}_{2}=\mathrm{H} & \mathrm{R}_{3}=\mathrm{H} & \mathrm{R}_{4}=\mathrm{H} & \mathrm{R}_{5}=\mathrm{OH} & \mathrm{R}_{6}=\mathrm{MeO} \\ \text { Oleracein C } & \left(\mathrm{C}_{30} \mathrm{H}_{35} \mathrm{NO}_{16} ; 664\right) & \mathrm{R}_{1}=\mathrm{Glc} & \mathrm{R}_{2}=\mathrm{H} & \mathrm{R}_{3}=\mathrm{H} & \mathrm{R}_{4}=\mathrm{H} & \mathrm{R}_{5}=\mathrm{O}-\mathrm{Glc} & \mathrm{R}_{6}=\mathrm{H} \\ \text { Oleracein D } & \left(\mathrm{C}_{31} \mathrm{H}_{37} \mathrm{NO}_{17} ; 694\right) & \mathrm{R}_{1}=\mathrm{Glc} & \mathrm{R}_{2}=\mathrm{H} & \mathrm{R}_{3}=\mathrm{H} & \mathrm{R}_{4}=\mathrm{H} & \mathrm{R}_{5}=\mathrm{O}-\mathrm{Glc} & \mathrm{R}_{6}=\mathrm{MeO} \\ \text { Oleracein F } & \left(\mathrm{C}_{26} \mathrm{H}_{29} \mathrm{NO}_{12} ; 546\right) & \mathrm{R}_{1}=\mathrm{Glc} & \mathrm{R}_{2}=\mathrm{Me} & \mathrm{R}_{3}=\mathrm{H} & \mathrm{R}_{4}=\mathrm{MeO} & \mathrm{R}_{5}=\mathrm{OH} & \mathrm{R}_{6}=\mathrm{H} \\ \text { Oleracein G } & \left(\mathrm{C}_{25} \mathrm{H}_{27} \mathrm{NO}_{11} ; 516\right) & \mathrm{R}_{1}=\mathrm{Glc} & \mathrm{R}_{2}=\mathrm{Me} & \mathrm{R}_{3}=\mathrm{H} & \mathrm{R}_{4}=\mathrm{H} & \mathrm{R}_{5}=\mathrm{OH} & \mathrm{R}_{6}=\mathrm{H} \\ \text { Oleracein T } & \left(\mathrm{C}_{27} \mathrm{H}_{21} \mathrm{NO}_{5} ; 486\right) & \mathrm{R}_{1}=\mathrm{Cou} & \mathrm{R}_{2}=\mathrm{H} & \mathrm{R}_{3}=\mathrm{H} & \mathrm{R}_{4}=\mathrm{OH} & \mathrm{R}_{5}=\mathrm{H} & \mathrm{R}_{6}=\mathrm{H} \\ \text { Oleracein U } & \left(\mathrm{C}_{15} \mathrm{H}_{15} \mathrm{NO}_{6} ; 340\right) & \mathrm{R}_{1}=\mathrm{H} & \mathrm{R}_{2}=\mathrm{H} & \mathrm{R}_{3}=\mathrm{H} & \mathrm{R}_{4}=\mathrm{OH} & \mathrm{R}_{5}=\mathrm{H} & \mathrm{R}_{6}=\mathrm{H} \\ \text { Oleracein V } & \left(\mathrm{C}_{24} \mathrm{H}_{25} \mathrm{NO}_{13} ; 534\right) & \mathrm{R}_{1}=\mathrm{Glc} & \mathrm{R}_{2}=\mathrm{H} & \mathrm{R}_{3}=\mathrm{OH} & \mathrm{R}_{4}=\mathrm{OH} & \mathrm{R}_{5}=\mathrm{OH} & \mathrm{R}_{6}=\mathrm{H} \\ \text { Oleracein W } & \left(\mathrm{C}_{15} \mathrm{H}_{15} \mathrm{NO}_{17} ; 356\right) & \mathrm{R}_{1}=\mathrm{H} & \mathrm{R}_{2}=\mathrm{H} & \mathrm{R}_{3}=\mathrm{H} & \mathrm{R}_{4}=\mathrm{OH} & \mathrm{R}_{5}=\mathrm{OH} & \mathrm{R}_{6}=\mathrm{H} \\ \text { Oleracein Y } & \left(\mathrm{C}_{24} \mathrm{H}_{25} \mathrm{NO}_{12} ; 518\right) & \mathrm{R}_{1}=\mathrm{Glc} & \mathrm{R}_{2}=\mathrm{H} & \mathrm{R}_{3}=\mathrm{H} & \mathrm{R}_{4}=\mathrm{OH} & \mathrm{R}_{5}=\mathrm{OH} & \mathrm{R}_{6}=\mathrm{H}\end{array}$

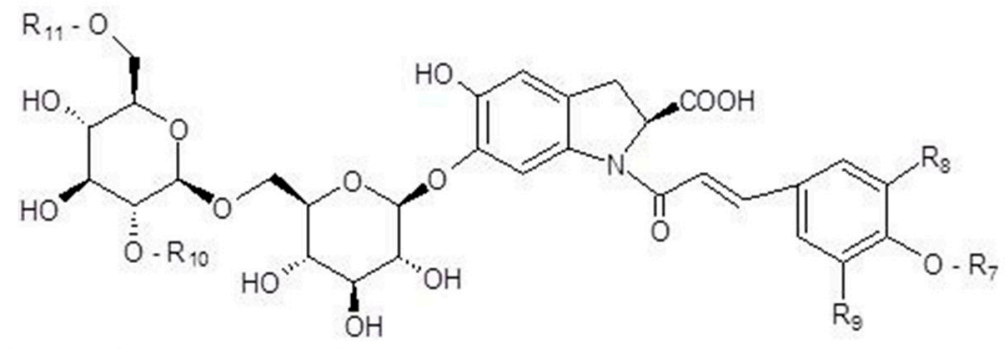

$\begin{array}{lllllll}\text { Oleracein H } & \left(\mathrm{C}_{30} \mathrm{H}_{35} \mathrm{NO}_{16} ; 664\right) & \mathrm{R}_{7}=\mathrm{H} & \mathrm{R}_{8}=\mathrm{H} & \mathrm{R}_{9}=\mathrm{H} & \mathrm{R}_{10}=\mathrm{H} & \mathrm{R}_{11}=\mathrm{H} \\ \text { Oleracein I } & \left(\mathrm{C}_{31} \mathrm{H}_{37} \mathrm{NO}_{17} ; 694\right) & \mathrm{R}_{7}=\mathrm{H} & \mathrm{R}_{8}=\mathrm{H} & \mathrm{R}_{9}=\mathrm{MeO} & \mathrm{R}_{10}=\mathrm{H} & \mathrm{R}_{11}=\mathrm{H} \\ \text { Oleracein J } & \left(\mathrm{C}_{40} \mathrm{H}_{43} \mathrm{NO}_{20} ; 856\right) & \mathrm{R}_{7}=\mathrm{H} & \mathrm{R}_{8}=\mathrm{OH} & \mathrm{R}_{9}=\mathrm{H} & \mathrm{R}_{10}=\mathrm{H} & \mathrm{R}_{11}=\mathrm{Fer} \\ \text { Oleracein K } & \left(\mathrm{C}_{39} \mathrm{H}_{41} \mathrm{NO}_{19} ; 826\right) & \mathrm{R}_{7}=\mathrm{H} & \mathrm{R}_{8}=\mathrm{H} & \mathrm{R}_{9}=\mathrm{H} & \mathrm{R}_{10}=\mathrm{Caf} & \mathrm{R}_{11}=\mathrm{H} \\ \text { Oleracein L } & \left(\mathrm{C}_{40} \mathrm{H}_{43} \mathrm{NO}_{20} ; 856\right) & \mathrm{R}_{7}=\mathrm{H} & \mathrm{R}_{8}=\mathrm{MeO} & \mathrm{R}_{9}=\mathrm{H} & \mathrm{R}_{10}=\mathrm{Caf} & \mathrm{R}_{11}=\mathrm{H} \\ \text { Oleracein M } & \left(\mathrm{C}_{41} \mathrm{H}_{45} \mathrm{NO}_{20} ; 870\right) & \mathrm{R}_{7}=\mathrm{H} & \mathrm{R}_{8}=\mathrm{H} & \mathrm{R}_{9}=\mathrm{H} & \mathrm{R}_{10}=\mathrm{H} & \mathrm{R}_{11}=\mathrm{Sin} \\ \text { Oleracein N } & \left(\mathrm{C}_{40} \mathrm{H}_{43} \mathrm{NO}_{19} ; 840\right) & \mathrm{R}_{7}=\mathrm{H} & \mathrm{R}_{8}=\mathrm{H} & \mathrm{R}_{9}=\mathrm{H} & \mathrm{R}_{10}=\mathrm{Fer} & \mathrm{R}_{11}=\mathrm{H} \\ \text { Oleracein O } & \left(\mathrm{C}_{41} \mathrm{H}_{45} \mathrm{NO}_{20} ; 870\right) & \mathrm{R}_{7}=\mathrm{H} & \mathrm{R}_{8}=\mathrm{H} & \mathrm{R}_{9}=\mathrm{MeO} & \mathrm{R}_{10}=\mathrm{Fer} & \mathrm{R}_{11}=\mathrm{H} \\ \text { Oleracein P } & \left(\mathrm{C}_{36} \mathrm{H}_{45} \mathrm{NO}_{21} ; 826\right) & \mathrm{R}_{7}=\mathrm{Glc} & \mathrm{R}_{8}=\mathrm{H} & \mathrm{R}_{9}=\mathrm{H} & \mathrm{R}_{10}=\mathrm{H} & \mathrm{R}_{11}=\mathrm{H} \\ \text { Oleracein Q } & \left(\mathrm{C}_{37} \mathrm{H}_{47} \mathrm{NO}_{22} ; 856\right) & \mathrm{R}_{7}=\mathrm{Glc} & \mathrm{R}_{8}=\mathrm{MeO} & \mathrm{R}_{9}=\mathrm{H} & \mathrm{R}_{10}=\mathrm{H} & \mathrm{R}_{11}=\mathrm{H} \\ \text { Oleracein R } & \left(\mathrm{C}_{40} \mathrm{H}_{53} \mathrm{NO}_{24} ; 1002\right) & \mathrm{R}_{7}=\mathrm{Glc} & \mathrm{R}_{8}=\mathrm{H} & \mathrm{R}_{9}=\mathrm{H} & \mathrm{R}_{10}=\mathrm{Fer} & \mathrm{R}_{11}=\mathrm{H} \\ \text { Oleracein S } & \left(\mathrm{C}_{40} \mathrm{H}_{43} \mathrm{NO}_{19} ; 840\right) & \mathrm{R}_{7}=\mathrm{H} & \mathrm{R}_{8}=\mathrm{H} & \mathrm{R}_{9}=\mathrm{H} & \mathrm{R}_{10}=\mathrm{H} & \mathrm{R}_{11}=\mathrm{Fer} \\ \text { Oleracein X } & \left(\mathrm{C}_{30} \mathrm{H}_{35} \mathrm{NO}_{17} ; 680\right) & \mathrm{R}_{7}=\mathrm{H} & \mathrm{R}_{8}=\mathrm{OH} & \mathrm{R}_{9}=\mathrm{H} & \mathrm{R}_{10}=\mathrm{H} & \mathrm{R}_{11}=\mathrm{H}\end{array}$<smiles>O=C1CCC2c3cc(O)c(O)cc3CCN12</smiles>

Oleracein $\mathrm{E}\left(\mathrm{C}_{12} \mathrm{H}_{13} \mathrm{NO}_{3} ; 218\right)$

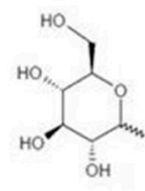

Glucosyl (Glc)

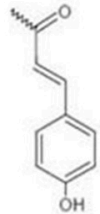

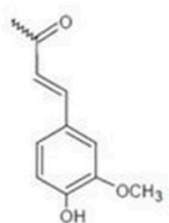

eruloyl (Fer)

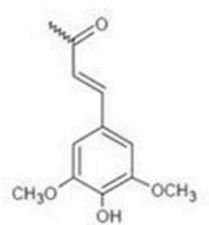

Sinapoyl (Sin)

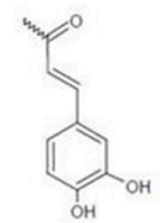

Caffeoyl (Caf)

Figure 5. Chemical structures and molecular ions $[\mathrm{M}-\mathrm{H}]^{-}$of oleraceins A-Y. 


\subsubsection{Other Compounds}

Compounds $\mathbf{1}$ and $\mathbf{2}$ were organic acids, showing the same deprotonated molecular ions. They were characterized as isocitric acid and citric acid, respectively, by comparison with an analytical standard of citric acid. Compound 3, with $[\mathrm{M}-\mathrm{H}]^{-}$at $\mathrm{m} / \mathrm{z} 315$, and fragment ions at $m / z 153$ and 123, was named as hydroxytyrosol hexoside [34].

\subsection{Quantification of Phenolic Compounds in All Extracts}

Fifteen compounds were quantified in the analyzed extracts of Portulaca oleracea by HPLC, measuring phenolic acids at $320 \mathrm{~nm}$, flavonoids at $350 \mathrm{~nm}$, and epicatechin and hydroxytyrosol hexoside at $280 \mathrm{~nm}$. Citric acid was quantified in MS/MS mode with an analytical standard (Table 2).

Table 2. Quantification of compounds in raw and steamed extracts of Portulaca oleracea.

\begin{tabular}{|c|c|c|c|c|}
\hline & $N^{\circ}$. & Raw & Steamed & Loss $(\%)$ \\
\hline \multicolumn{5}{|c|}{ Phenolic Acids } \\
\hline 4 & Caffeoylglucaric acid & $18.1 \pm 0.6^{\mathrm{a}}$ & $16.4 \pm 0.7^{\mathrm{b}}$ & $9.4 \pm 0.9$ \\
\hline 5 & Caffeic acid glucuronide isomer & $14.30 \pm 0.08^{\mathrm{a}}$ & $12.3 \pm 0.4^{b}$ & $14 \pm 2$ \\
\hline 6 & Caffeic acid-O-hexoside & $14.12 \pm 0.08^{\mathrm{a}}$ & $12.9 \pm 0.7^{b}$ & $9 \pm 4$ \\
\hline 7 & Caffeic acid glucuronide isomer & $27.8 \pm 0.7^{\mathrm{a}}$ & $23 \pm 1^{b}$ & $17 \pm 2$ \\
\hline 9 & Ferulic acid-O-hexoside & $9.3 \pm 0.5^{\mathrm{a}}$ & $7.7 \pm 0.2^{b}$ & $17 \pm 2$ \\
\hline 11 & Sinapic acid-O-hexoside & $38.0 \pm 0.4^{\mathrm{a}}$ & $32 \pm 1^{b}$ & $16 \pm 2$ \\
\hline 17 & Ferulic acid derivative & $18.44 \pm 0.03^{\mathrm{a}}$ & $15 \pm 1^{b}$ & $19 \pm 5$ \\
\hline \multirow{2}{*}{\multicolumn{5}{|c|}{$\begin{array}{l}\text { Total } \\
\text { Flavonoids }\end{array}$}} \\
\hline & & & & \\
\hline 12 & Epicatechin & $28 \pm 2^{a}$ & $30 \pm 3^{a}$ & - \\
\hline 18 & Quercetin-O-hexoside isomer & $13.2 \pm 0.3^{\mathrm{a}}$ & $11.2 \pm 0.7^{\mathrm{b}}$ & $15 \pm 3$ \\
\hline 22 & Kaempferol-O-hexoside & $15 \pm 1^{\mathrm{a}}$ & $13.6 \pm 0.9^{\mathrm{a}}$ & $9.0 \pm 0.1$ \\
\hline 23 & Isorhamnetin-O-hexoside & $15.9 \pm 0.2^{\mathrm{a}}$ & $13 \pm 1^{b}$ & $18 \pm 5$ \\
\hline 24 & Quercetin-O-hexoside isomer & $18.4 \pm 0.7^{\mathrm{a}}$ & $13.8 \pm 0.7^{b}$ & $25 \pm 1$ \\
\hline Total & & $91 \pm 2^{a}$ & $82 \pm 3^{b}$ & $10 \pm 1$ \\
\hline \multicolumn{5}{|c|}{ Other compounds } \\
\hline 1 & Isocitric acid & $550 \pm 40^{\mathrm{a}}$ & $500 \pm 40^{\mathrm{a}}$ & $9.1 \pm 0.7$ \\
\hline 2 & Citric acid & $600 \pm 50^{\mathrm{a}}$ & $440 \pm 20^{b}$ & $26 \pm 3$ \\
\hline 3 & Hydroxytyrosol hexoside & $3.89 \pm 0.02^{\mathrm{a}}$ & $3.83 \pm 0.08^{\mathrm{a}}$ & $2 \pm 1$ \\
\hline Total & & $1150 \pm 60^{a}$ & $940 \pm 40^{\mathrm{b}}$ & $18.2 \pm 0.8$ \\
\hline TIPC & & $1380 \pm 60^{a}$ & $1140 \pm 40^{b}$ & $17.4 \pm 0.7$ \\
\hline
\end{tabular}

Values (mg/100 g DE) are mean \pm SD of three parallel measurements. Different superscripts $\left({ }^{\mathrm{a}}\right.$ and $\left.{ }^{\mathrm{b}}\right)$ indicate significant differences in the extracts $(p<0.05)$.

Oleraceins were not quantified because there are no commercial standards of these phenolic alkaloids. We made a heatmap with the values of the areas (\%) of each of the identified compounds with respect to total area, in order to visualize the contribution of each of the compounds (Figure 6) to the extracts. Areas were calculated for each compound using Extracted Ion Chromatograms at the corresponding deprotonated molecular ion. This figure shows that oleracein $\mathrm{A}$ is the major compound in both extracts, followed by isocitric acid, citric acid, oleracein $\mathrm{U}$, sinapic acid-O-hexoside and the new oleraceins $\mathrm{X}$ and Y. In previous works, oleracein A also appears as the major compound in Portulaca oleracea $[13,19]$. 


\begin{tabular}{|c|c|c|c|}
\hline № & Compound & Raw & Steamed \\
\hline 1 & Isocitric acid & 10.1 & 9.4 \\
\hline 2 & Citric acid & 6.9 & 7.9 \\
\hline 3 & Hydroxytyrosol hexoside & 1.0 & 1.1 \\
\hline 4 & Caffeoylglucaric acid & 0.9 & 0.9 \\
\hline 5 & Caffeic acid glucuronide isomer & 0.4 & 0.5 \\
\hline 6 & Caffeic acid-O-hexoside & 1.3 & 1.2 \\
\hline 7 & Caffeic acid glucuronide isomer & 2.8 & 2.9 \\
\hline 8 & Catechin & 1.4 & 1.6 \\
\hline 9 & Ferulic acid-O-hexoside & 0.5 & 0.7 \\
\hline 10 & Oleracein C & 1.3 & 0.8 \\
\hline 11 & Sinapic acid- $O$-hexoside & 5.5 & 6.8 \\
\hline 12 & Epicatechin & 1.7 & 2.4 \\
\hline 13 & Oleracein X & 6.2 & 4.9 \\
\hline 14 & Oleracein Y & 6.2 & 6.6 \\
\hline 15 & Oleracein A & 29.8 & 27.4 \\
\hline 16 & Oleracein B & 2.0 & 1.4 \\
\hline 17 & Ferulic acid derivative & 0.8 & 0.5 \\
\hline 18 & Quercetin-O-hexoside isomer & 1.1 & 0.7 \\
\hline 19 & Oleracein $\mathbf{N}$ & 0.5 & 0.1 \\
\hline 20 & Oleracein U & 6.9 & 7.8 \\
\hline 21 & Oleracein J & 0.7 & 0.6 \\
\hline 22 & Kaempferol-O-hexoside & 1.2 & 1.7 \\
\hline 23 & Isorhamnetin-O-hexoside & 2.3 & 1.8 \\
\hline 24 & Quercetin-O-hexoside isomer & 2.8 & 1.8 \\
\hline 25 & Unknown & 5.6 & 8.6 \\
\hline & Lower concentration & \multicolumn{2}{|c|}{ Higher concentration } \\
\hline
\end{tabular}

Figure 6. Relative peak areas and heat map of the raw and steamed extracts of Portulaca oleracea aerial parts.

From a qualitative point of view, raw and steamed extracts presented the same phenolic profile, although higher concentrations of phenolic acids, flavonoids and other compounds were observed in raw Portulaca, being TIPC (Total Individual Phenolic Content, defined as the sum of all individual phenolic concentrations) values of $1380 \mathrm{mg} / 100 \mathrm{~g}$ DE and $1140 \mathrm{mg} / 100 \mathrm{~g} \mathrm{DE}$ in raw and steamed samples, respectively. It was thus observed the initial hypothesis of a decrease in bioactive compounds during the steaming process was confirmed. However, the decrease in TIPC was lower than $20 \%$. The losses of each individual compound after the steaming process are given in Table 2, observing that the concentration of most compounds, except epicatechin, decreased approximately between 10 and $20 \%$.

Isocitric acid and citric acid were the most abundant compounds in all extracts, presenting values of 550 and $600 \mathrm{mg} / 100 \mathrm{~g}$ DE for raw extract, and 500 and $440 \mathrm{mg} / 100 \mathrm{~g}$ $\mathrm{DE}$ for steamed extract. Both compounds represented in raw and steamed extracts more than $80 \%$ of the TIPC. Citric acid has been reported in Portulaca oleracea in the range of 1655- $\mathrm{mg} / 100 \mathrm{~g}$ DE [41]. Citric acid is commonly used in the food, pharmaceutical, and cosmetic industries. In addition to being a flavor enhancing additive, this compound has been reported to behave as an antioxidant [42].

Sinapic acid-O-hexoside was the phenolic acid found in greater quantity, reaching values of $38 \mathrm{mg} / 100 \mathrm{~g}$ DE and $32 \mathrm{mg} / 100 \mathrm{~g}$ DE in raw and steamed extracts, respectively, 
which meant $27 \%$ of the total phenolic acids in both cases. A value of $22.1 \mathrm{mg} / 100 \mathrm{~g} \mathrm{DE}$ was previously reported in literature for this plant [11].

Aerial parts of Portulaca oleracea were also rich in flavonoids such as those derived from kaempferol, quercetin, and epicatechin, the latter being the only one of all quantified phenols that had a higher value in the steamed extract $(30 \mathrm{mg} / 100 \mathrm{~g} \mathrm{DE})$ than in the raw extract $(28 \mathrm{mg} / 100 \mathrm{~g} \mathrm{DE})$, which respectively represented $37 \%$ and $31 \%$ of the total flavonoids. In previous works, flavonoids (derivatives of kaempferol and quercetin, among others) were also reported in P. oleracea $[12,13,43]$.

\subsection{Total Phenolic Content and Antioxidant Activity}

Spectrophotometric methods are still frequently used to determine total phenolic content, as well as to report the antioxidant activity of plant extracts. In our opinion, their simplicity, low-cost, and possibility to compare with previous research makes them very useful. However, it is important to consider that the data obtained by these assays are preliminary (mainly screening methods), and the use of HPLC-MS (or similar techniques) is mandatory for the identification and quantitation of phytochemicals. Each antioxidant assay presents a different mechanism and specific handicaps that need to be taking into account for a proper interpretation of the results [44].

The results of the TPC assay for raw and steamed Portulaca oleracea extracts are given in Figure 7. In accordance with HPLC-MS results, the raw extract possessed a higher concentration of phenolic content (57 mg GAE/g DE) than the steamed extract (33 mg GAE/g DE), showing a statistically significant difference between their values. In previous literature, total bioactive components for raw Portulaca oleracea have been reported, with maximum values between 3.6 and $13.4 \mathrm{mg}$ GAE/g DE [14,18,25-27]. In a previous work comparing the raw, boiled, and blanched P. oleracea, it was observed that the raw sample had a higher TPC value (23 mg GAE/g DE) compared to the values of the cooked samples (boiled $19 \mathrm{mg}$ GAE/g DE and blanched $10 \mathrm{mg} \mathrm{GAE} / \mathrm{g}$ DE) [23]. In another study, Naser Aldeen et al. obtained slightly higher values for cooked than raw purslane (13.13 mg GAE/g DE and $12.75 \mathrm{mg} \mathrm{GAE/g} \mathrm{DE,} \mathrm{respectively)} \mathrm{[24].}$

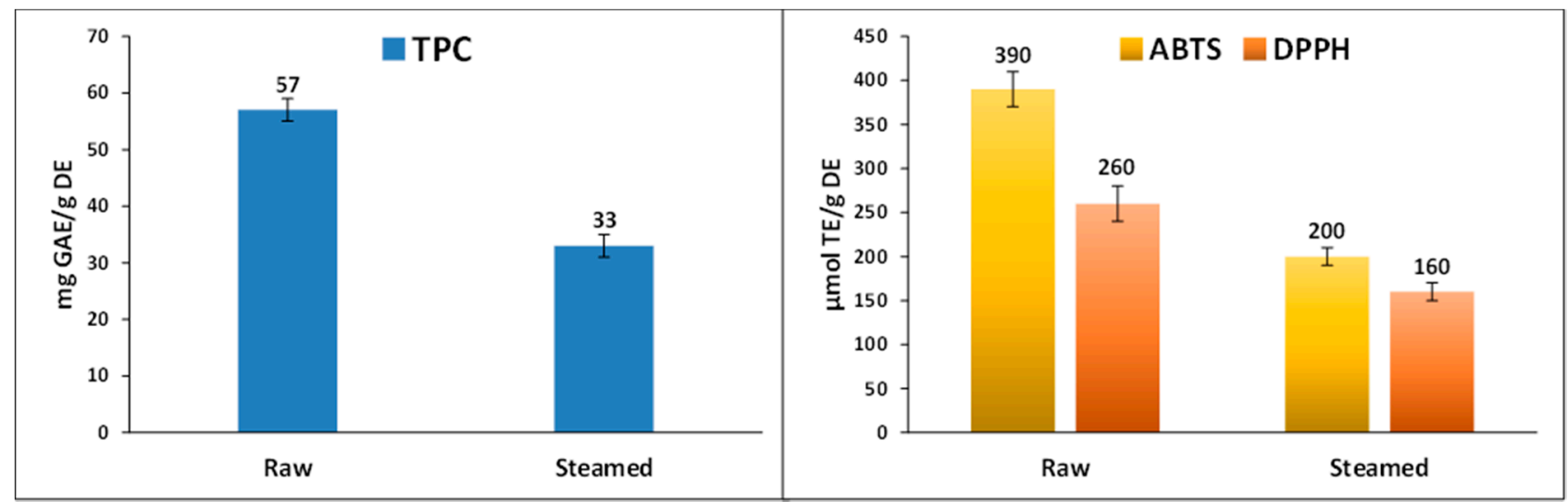

Figure 7. Total phenolic content (TPC) and antioxidant assays (ABTS and DPPH) of raw and steamed Portulaca oleracea aerial parts. Values are given as mean \pm standard deviation of three parallel measurements. GAE: Gallic acid equivalent; TE: Trolox equivalent; DE: Dried extract.

The antioxidant capacity of Portulaca oleracea extracts was also evaluated; results are summarized in Figure 7. The study of the antioxidant capacity of plant extracts is of great interest in order to provide new and safer natural antioxidants for pharmaceutical and food industries. So, in this work, free radical quenching (ABTS ${ }^{+}$and DPPH assays) was studied to examine the antioxidant effects of raw and steamed Portulaca oleracea extracts. In both assays, the antioxidant effects showed a similar trend to total bioactive compounds, decreasing after the steaming process, in agreement with the initial hypothesis of the work. 
The values for ABTS ${ }^{+}$and DPPH assays of the raw extract were $390 \mu \mathrm{mol}$ TE/g DE and $260 \mu \mathrm{mol} \mathrm{TE} / \mathrm{g} \mathrm{DE}$, and for the steamed extract were $200 \mu \mathrm{mol} \mathrm{TE} / \mathrm{g}$ DE and $160 \mu \mathrm{mol}$ $\mathrm{TE} / \mathrm{g} \mathrm{DE}$, so the raw extract was more active on free radicals than the steamed extract. However, it can be observed that a higher decrease was observed in ABTS values with respect to DPPH ones. A previously reported value in an ABTS assay for raw purslane was $102.7 \mu \mathrm{mol} \mathrm{TE} / \mathrm{g}$ DE, lower than that obtained in our study [43]. In another work on aerial parts of Portulaca oleracea, the cooking process with lemon juice as additive increased the antioxidant activity compared to raw samples in the DPPH assay [24]. The decrease in antioxidant activity observed after the steaming process is in agreement with the decrease in phenolic content (Table 2). Considering that the decrease of concentration was similar in all compounds (as well as TIPC), the lower antioxidant activity in steamed samples cannot be attributed to particular compounds. Although the observed antioxidant capacity (in both raw and steamed samples) was mainly due to the most abundant compounds, the whole extract must be considered to account for the antioxidant potency.

\section{Conclusions}

In this work, we have reported the phenolic profile and antioxidant activity of aerial parts of Portulaca oleracea L. The most abundant compounds were oleraceins, followed by citric and isocitric acids. In addition, the influence of the steaming cooking process has been reported for the first time. The initial hypothesis of a decrease in both the concentration of bioactive compounds and the antioxidant capacity was confirmed by HPL-MS (quantification of phytochemicals) and antioxidant assays; the decrease in phenolic concentration and antioxidant activity after the steaming process were in agreement. However, it is important to mention that the steamed samples still contained a significant amount of the initial compounds and kept a considerable antioxidant activity. We also identified two new oleraceins that have not been previously reported in scientific literature. Their structures were elucidated by ion-trap mass spectrometry and confirmed by exact mass using Q-TOF.

Author Contributions: Methodology, M.d.P.F.-P., E.J.L.-M. and A.R.-M.; investigation, M.d.P.F.-P.; formal analysis, M.d.P.F.-P.; data curation, M.d.P.F.-P. and E.J.L.-M.; writing—original draft, M.d.P.F.-P.; writing-review and editing, E.J.L.-M. and A.R.-M.; conceptualization, E.J.L.-M. and A.R.-M.; supervision, E.J.L.-M. and A.R.-M. All authors have read and agreed to the published version of the manuscript.

Funding: This research received no external funding.

Institutional Review Board Statement: Not applicable.

Informed Consent Statement: Not applicable.

Data Availability Statement: Data available on request.

Acknowledgments: Technical and human support provided by CICT of Universidad de Jaén (UJA, MINECO, Junta de Andalucía, FEDER) is gratefully acknowledged.

Conflicts of Interest: The authors declare no conflict of interest.

\section{References}

1. Jiménez-Aguilar, D.M.; Grusak, M.A. Minerals, vitamin C, phenolics, flavonoids and antioxidant activity of Amaranthus leafy vegetables. J. Food Compos. Anal. 2017, 58, 33-39. [CrossRef]

2. Durazzo, A.; Lisciani, S.; Camilli, E.; Gabrielli, P.; Marconi, S.; Gambelli, L.; Aguzzi, A.; Lucarini, M.; Maiani, G.; Casale, G.; et al. Nutritional composition and antioxidant properties of traditional Italian dishes. Food Chem. 2017, 218, 70-77. [CrossRef]

3. Sergio, L.; Boari, F.; Pieralice, M.; Linsalata, V.; Cantore, V.; Di Venere, D. Bioactive phenolics and antioxidant capacity of some wild edible greens as affected by different cooking treatments. Foods 2020, 9, 1320. [CrossRef]

4. Petropoulos, S.; Karkanis, A.; Martins, N.; Ferreira, I.C.F.R. Phytochemical composition and bioactive compounds of common purslane (Portulaca oleracea L.) as affected by crop management practices. Trends Food Sci. Technol. 2016, 55, 1-10. [CrossRef]

5. Uddin, M.K.; Juraimi, A.S.; Hossain, M.S.; Nahar, M.A.U.; Ali, M.E.; Rahman, M.M. Purslane weed (Portulaca oleracea): A prospective plant source of nutrition, omega-3 fatty acid, and antioxidant attributes. Sci. World J. 2014, 2014, 951019. [CrossRef] [PubMed] 
6. Xu, X.; Yu, L.; Chen, G. Determination of flavonoids in Portulaca oleracea L. by capillary electrophoresis with electrochemical detection. J. Pharm. Biomed. Anal. 2006, 41, 493-499. [CrossRef] [PubMed]

7. Chan, K.; Islam, M.W.; Kamil, M.; Radhakrishnan, R.; Zakaria, M.N.M.; Habibullah, M.; Attas, A. The analgesic and antiinflammatory effects of Portulaca oleracea L. subsp. sativa (Haw.) Celak. . J. Ethnopharmacol. 2000, 73, 445-451. [CrossRef]

8. Zidan, Y.; Bouderbala, S.; Djellouuli, F.; Lacaille-Dubois, M.A.; Bouchenak, M. Portulaca oleracea reduces triglyceridemia, cholesterolemia, and improves lecithin: Cholesterol acyltransferase activity in rats fed enriched-cholesterol diet. Phytomedicine 2014, 21, 1504-1508. [CrossRef] [PubMed]

9. Lee, A.S.; Kim, J.S.; Lee, Y.J.; Kang, D.G.; Lee, H.S. Anti-TNF- $\alpha$ activity of Portulaca oleracea in vascular endothelial cells. Int. J. Mol. Sci. 2012, 13, 5628-5644. [CrossRef] [PubMed]

10. Filannino, P.; Di Cagno, R.; Trani, A.; Cantatore, V.; Gambacorta, G.; Gobbetti, M. Lactic acid fermentation enriches the profile of biogenic compounds and enhances the functional features of common purslane (Portulaca oleracea L.). J. Funct. Foods 2017, 39, 175-185. [CrossRef]

11. Petropoulos, S.A.; Fernandes, Â.; Dias, M.I.; Vasilakoglou, I.B.; Petrotos, K.; Barros, L.; Ferreira, I.C.F.R. Nutritional value, chemical composition and cytotoxic properties of common purslane (Portulaca oleracea L.) in relation to harvesting stage and plant part. Antioxidants 2019, 8, 293. [CrossRef] [PubMed]

12. Farag, M.A.; Abdel Shakour, Z.T. Metabolomics driven analysis of 11 Portulaca leaf taxa as analysed via UPLC-ESI-MS/MS and chemometrics. Phytochemistry 2019, 161, 117-129. [CrossRef] [PubMed]

13. Voynikov, Y.; Gevrenova, R.; Balabanova, V.; Doytchinova, I.; Nedialkov, P.; Zheleva-Dimitrova, D. LC-MS analysis of phenolic compounds and oleraceins in aerial parts of Portulaca oleracea L. J. Appl. Bot. Food Qual. 2019, 92, 298-312. [CrossRef]

14. Alam, A.; Juraimi, A.S.; Rafii, M.Y.; Hamid, A.A.; Aslani, F.; Hasan, M.M.; Zainudin, M.A.M.; Uddin, K. Evalutation of antioxidant compounds, antioxidant activities, and mineral composition of 13 collected purslane (Portulaca oleracea L.) accessions. BioMed Res. Int. 2014. [CrossRef]

15. Turan, M.; Kordali, S.; Zengin, H.; Dursun, A.; Sezen, Y. Macro and micro mineral content of some wild edible leaves consumed in Eastern Anatolia. Acta Agric. Scand. Sect. B Plant Sci. 2003, 53, 129-137. [CrossRef]

16. Sicari, V.; Loizzo, M.R.; Tundis, R.; Mincione, A.; Pellicano, T.M. Portulaca oleracea L. (Purslane) extracts display antioxidant and hypoglycaemic effects. J. Appl. Bot. Food Qual. 2018, 91, 39-46. [CrossRef]

17. Nemzer, B.; Al-Taher, F.; Abshiru, N. Phytochemical composition and nutritional value of different plant parts in two cultivated and wild purslane (Portulaca oleracea L.) genotypes. Food Chem. 2020, 320, 126621. [CrossRef]

18. Alu'datt, M.H.; Rababah, T.; Alhamad, M.N.; Al-Tawaha, A.; Al-Tawaha, A.R.; Gammoh, S.; Ereifej, K.I.; Al-Karaki, G.; Hamasha, H.R.; Tranchant, C.C.; et al. Herbal yield, nutritive composition, phenolic contents and antioxidant activity of purslane (Portulaca oleracea L.) grown in different soilless media in a closed system. Ind. Crops Prod. 2019, 141, 111746. [CrossRef]

19. Jiao, Z.; Wang, H.; Wang, P.; Sun, H.; Yue, S.; Xiang, L. Detection and quantification of cyclo-dopa amides in Portulaca oleracea L. by HPLC-DAD and HPLC-ESI-MS/MS. J. Chin. Pharm. Sci. 2014, 23, 533-542. [CrossRef]

20. Wang, P.; Sun, H.; Liu, D.; Jiao, Z.; Yue, S.; He, X.; Xia, W.; Ji, J.; Xiang, L. Protective effect of a phenolic extrac containing indoline amides from Portulaca oleracea against congnitive impairment in senescent mice induced by large dose of $\mathrm{D}$-galactose// $\mathrm{NaNO}$. J. Ethnopharmacol. 2017, 203, 252-259. [CrossRef]

21. Moreno, D.A.; Carvajal, M.; López-Berenguer, C.; García-Viguera, C. Chemical and biological characterisation of nutraceutical compounds of broccoli. J. Pharm. Biomed. Anal. 2006, 41, 1508-1522. [CrossRef] [PubMed]

22. Agte, V.V.; Tarwadi, K.V.; Mengale, S.; Chiplonkar, S.A. Potential of traditionally cooked green leafe vegetables as natural sources for supplementation of eight micronutrients in vegetarian diets. J. Food Compos. Anal. 2000, 13, 885-891. [CrossRef]

23. Nagarani, G.; Abirami, A.; Nikitha, P.; Siddhuraju, P. Effect of hydrothermal processing on total polyphenolics and antioxidant potential of underutilized leafy vegetables, Boerhaavia diffusa and Portulaca oleracea. Asian Pac. J. Trop. Biomed. 2014, 4, S468-S477. [CrossRef] [PubMed]

24. Naser Aldeen, M.G.; Mansour, R.; AlJoubbeh, M. The effect of food additives and cooking on the antioxidant properties of purslane. Nutr. Food Sci. 2019, 50, 13-20. [CrossRef]

25. Lim, Y.Y.; Quah, E.P.L. Antioxidant properties of different cultivars of Portulaca oleracea. Food Chem. 2007, 103, 734-740. [CrossRef]

26. Uddin, K.; Juraimi, A.S.; Ali, E.; Ismail, M.R. Evaluation of antioxidant properties and mineral composition of Purslane (Portulaca oleracea L.) at different growth stages. Int. J. Mol. Sci. 2012, 13, 10257-10267. [CrossRef]

27. Karoune, S.; Kechebar, M.S.A.; Douffi, H.; Djellouli, A. Phenolic compounds and their antioxidant activities in Portulaca oleracea L. related to solvent extraction. Int. J. Biosci. 2017, 11, 147-155. [CrossRef]

28. Fernández-Poyatos, M.D.P.; Ruiz-Medina, A.; Zengin, G.; Llorent-Martínez, E.J. Phenolic characterization, antioxidant activity, and enzyme inhibitory properties of Berberis thunbergii DC. leaves: A valuable source of phenolic acids. Molecules 2019, $24,4171$. [CrossRef]

29. Spínola, V.; Llorent-Martínez, E.J.; Gouveia, S.; Castilho, P.C. Myrica faya: A new source of antioxidant phytochemicals. J. Agric. Food Chem. 2014, 62, 9722-9735. [CrossRef]

30. Dini, I.; Tenore, G.C.; Dini, A. Effect of industrial and domestic processing on antioxidant properties of pumpkin pulp. LWT Food Sci. Technol. 2013, 53, 382-385. [CrossRef] 
31. Volden, J.; Borge, G.I.A.; Hansen, M.; Wicklund, T.; Bengtsson, G.B. Processing (blanching, boiling, steaming) effects on the content of glucosinolates and antioxidant-related parameters in cauliflower (Brassica oleracea L. ssp. botrytis). LWT Food Sci. Technol. 2009, 42, 63-73. [CrossRef]

32. Spínola, V.; Llorent-Martínez, E.J.; Gouveia-Figueira, S.; Castilho, P.C. Ulex europaeus: From noxious weed to source of valuable isoflavones and flavanones. Ind. Crops Prod. 2016, 90, 9-27. [CrossRef]

33. Steingass, C.B.; Glock, M.P.; Schweiggert, R.M.; Carle, R. Studies into the phenolic patterns of different tissues of pineapple

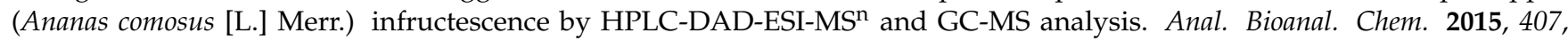
6463-6479. [CrossRef] [PubMed]

34. Llorent-Martínez, E.J.; Spínola, V.; Castilho, P.C. Phenolic profiles of Lauraceae plant species endemic to Laurisilva forest: A chemotaxonomic survey. Ind. Crops Prod. 2017, 107, 1-12. [CrossRef]

35. Llorent-Martínez, E.J.; Ortega-Barrales, P.; Zengin, G.; Mocan, A.; Simirgiotis, M.J.; Ceylan, R.; Uysal, S.; Aktumsek, A. Evaluation of antioxidant potential, enzyme inhibition activity and phenolic profile of Lathyrus cicera and Lathyrus digitatus: Potential sources of bioactive compounds for the food industry. Food Chem. Toxicol. 2017, 107, 609-619. [CrossRef] [PubMed]

36. Yang, Z.; Liu, C.; Xiang, L.; Zheng, Y. Phenolic alkaloids as a new class of antioxidants in Portulaca oleracea. Phyther. Res. 2009, 23, 1032-1035. [CrossRef]

37. Xiang, L.; Xing, D.; Wang, W.; Wang, R.; Ding, Y.; Du, L. Alkaloids from Portulaca oleracea L. Phytochemistry 2005, 66, $2595-2601$. [CrossRef]

38. Lin, W.; Ma, S. Enantioselective synthesis of naturally occurring isoquinoline alkaloids: (S)-(-)-trolline and (R)-(+)-oleracein E. Org. Chem. Front. 2017, 4, 958-966. [CrossRef]

39. Liu, D.; Shen, T.; Xiang, L. Two antioxidant alkaloids from Portulaca oleracea L. Helv. Chim. Acta 2011, 94, 497-501. [CrossRef]

40. Jiao, Z.Z.; Yue, S.; Sun, H.X.; Jin, T.Y.; Wang, H.N.; Zhu, R.X.; Xiang, L. Indoline amide glucosides from Portulaca oleracea: Isolation, structure, and DPPH scavenging activity. J. Nat. Prod. 2015, 78, 2588-2597. [CrossRef]

41. Oliveira, I.; Valentão, P.; Lopes, R.; Andrade, P.B.; Bento, A.; Pereira, J.A. Phytochemical characterization and radical scavenging activity of Portulaca oleraceae L. leaves and stems. Microchem. J. 2009, 92, 129-134. [CrossRef]

42. Ali, H.K.Q.; Daud, M.Z.M.; Al-Azzawi, Z. Economic benefit from the optimization of citric acid production from rice straw through Plackett-Burman design and central composite design. Turkish J. Eng. Environ. Sci. 2012, 36, 81-93. [CrossRef]

43. Cai, Y.; Luo, Q.; Sun, M.; Corke, H. Antioxidant activity and phenolic compounds of 112 traditional Chinese medicinal plants associated with anticancer. Life Sci. 2004, 74, 2157-2784. [CrossRef] [PubMed]

44. Granato, D.; Shahidi, F.; Wrolstad, R.; Kilmartin, P.; Melton, L.D.; Hidalgo, F.J.; Miyashita, K.; Van Camp, J.; Alasalvar, C.; Ismail, A.B.; et al. Antioxidant activity, total phenolics and flavonoids contents: Should we ban in vitro screening methods? Food Chem. 2018, 264, 471-475. [CrossRef] 